\title{
The power of a test for seasonality
}

\author{
S. D. WALTER
}

From the Department of Epidemiology and Public Health, Yale University School of Medicine, Connecticut, USA

SUMMARY The power of a test of Edwards to detect seasonality or cyclic variation in the rate of an epidemiological event is derived and tabulated. A simple harmonic or sinusoidal form of variation is assumed, but the power results should also apply approximately to other situations with similar types of rate oscillation. The results may be used by investigators to determine the sample size required in order to detect seasonal variation of a given magnitude, or conversely to calculate the probability of detecting seasonality of various amplitudes with a sample of fixed size.

An investigation of seasonality, or cyclic variation throughout the year, is a fairly common component of the basic aetiological description of diseases, particularly for congenital abnormalities, certain cancers of childhood, infectious diseases, and psychiatric disorders. The analysis of seasonal trends is also sometimes applied to the pattern of total births and total deaths in a population. A statistical test which has been widely used by epidemiologists is that of Edwards (1961); the only data required for this test are the numbers of cases of the disorder in question, grouped into appropriate time intervals such as months. Edwards's test ignores any possibility of variation in the population at risk which may itself be responsible for the apparent seasonality in the disease incidence; it also assumes that the time intervals are of equal length, which may not be true in practice (for example, the different lengths of the months of the year). Walter and Elwood (1975) generalised Edwards's test to circumvent these difficulties, making it possible, for example, to adjust for the varying frequency of total monthly births in an assessment of the seasonality of anencephalus (Elwood, 1975) or to allow for the fluctuating monthly pattern of total hospital admissions in an analysis of the seasonal trend in mania admissions (Symonds and Williams, 1976; Walter, 1977). Hewitt et al. (1971) have also proposed a nonparametric test based on the ordering of ranks for the monthly rates, and this is particularly useful when small sample sizes are encountered and the approximations to certain normal distributions in the Edwards and Walter-Elwood tests are poor. The Edwards and Walter-Elwood tests may easily be adapted to analyse cyclic variation with time periods other than a year (for example, variation between hours of the day or between days of the week); the Hewitt test could also be generalised in this way, although a tabulation of the distributions of the appropriate test statistic would be required if the number of time intervals was other than $\mathbf{1 2}$ (the problem for which the distribution was evaluated by Hewitt).

James (1976) has remarked that no results for the power of tests for seasonality are available, except by using power estimates for the usual $\chi^{2}$ test for homogeneity of rates; this will be weak in general because the usual $\chi^{2}$ test does not take into account the ordered structure of the seasonal variation. Power estimates are derived and tabulated here for the test of Edwards assuming a simple harmonic or sinusoidal model for the cyclic behaviour of the rates. The results should also be approximately correct for other similar models, and where the population at risk also fluctuates slightly.

\section{Method}

We suppose that in a particular time interval (for example, a month) there are $n$ cases of the disease in question (or births, etc), and that there are $\mathbf{k}$ equal time intervals making up the time unit of the study (for example, a year). The basic idea of the Edwards (1961) test is to represent the data by weights of magnitude $\sqrt{ } n$ placed on the circumference of a circle, with unit radius, which represents the year; thus the weight for a particular month is placed at an angle $\theta$ which corresponds to the midpoint of that month, taking a particular date (for example, 1 January) as the referent point $\theta=0^{\circ}$. Following convention, a pair of co-ordinate axes are defined with their origin at the centre of 
the circle, such that the positive $\mathrm{x}$-axis lies along $\theta=0^{\circ}$ and the positive $y$-axis lies along $\theta=90^{\circ}$ (see Walter and Elwood, 1975; Figure). The calculations required for Edwards's test are to find the observed position, in the $(x, y)$ co-ordinate system, of the centre of gravity of all the weights together, and also its expected position if the null hypothesis is true.

We denote by $\mathrm{N}=\Sigma \mathrm{n}$, the total sample size. If the null hypothesis (that of no seasonal trend, or equal underlying rates in each month) is correct, then the centre of gravity on the $\mathrm{x}$-axis, denoted by $\overline{\mathrm{x}}$, has an expected value zero and the variance is $\mathrm{V}(\overline{\mathbf{x}})=1 /(8 \mathrm{~N})($ Edwards, 1961); similarly $\mathrm{E}(\overline{\mathrm{y}})=0$ and $V(\bar{y})=1 /(8 N)$. This leads to a test statistic

$$
\mathrm{X}^{2}=8 \mathrm{~N}\left(\overline{\mathrm{x}}^{2}+\overline{\mathrm{y}}^{2}\right)
$$

which has approximately a $\chi^{2}$ distribution with 2 degrees of freedom in samples of moderate or large size. Thus using the conventional $5 \%$ significance level, this test will be significant if $\mathrm{X}^{2}$ is greater than 5.991 , the $5 \%$ point on the $\chi^{2}$ distribution with $2 \mathrm{df}$.

On the other hand, if seasonality does exist, then $\mathrm{X}^{2}$ will no longer have a $\chi^{2}$ distribution. If a particular kind of seasonal variation is postulated as an alternative hypothesis, and if the distribution of $\mathrm{X}^{2}$ under this alternative model can be determined, this will yield values for the power of the test against such an alternative; in other words it is then possible to find the probability that $\mathrm{X}^{2}$ is greater than 5.991 if the particular pattern of seasonality assumed under the alternative hypothesis is appropriate.

As it turns out, it is possible to determine the distribution of $\mathrm{X}^{2}$ if a sinusoidal or simple harmonic model is postulated for the rates. If the unique maximum of the sinusoidal rate curve occurs at the date with angle $\theta^{*}$, and if the proportional amplitude of variation from the average to the maximum is denoted by $a$ (for example, the value $a=0.1$ would imply a swing in rates of $10 \%$ from the mean in both directions during the year), then the rate on the day with angle $\theta$ is proportional to $\left[1+\mathrm{a} \operatorname{Cos}\left(\theta-\theta^{*}\right)\right]$.

Restricting our attention only to models with this sinusoidal form of variation has the advantage of relative simplicity in the algebraic derivation of the distribution of $\mathrm{X}^{2}$, but is not unduly limiting in the applicability of the ensuing power results. The harmonic model is one of the simplest which may be adopted to represent a gently oscillating rate, and it is easier to determine the parameters than in the case of other more erratic forms of variation. The power estimates tabulated below for the sinusoidal model should be applicable approximately to other models with the same general behaviour, even if they are not explicitly sinusoidal themselves.

We will assume here that the maximum rate occurs at $\theta^{*}=0^{\circ}$; there is no loss of generality in doing this because the actual value of $\theta^{*}$ has no effect on the power of the test; it is only the amplitude $a$ which is important in this context. Using this assumption it may be seen by symmetry that $E(\bar{y})=0$ as before. The expected value of $\bar{x}$, however, is given by taking moments along the $\mathrm{x}$-axis, giving

$$
\mathrm{E}(\overline{\mathrm{x}})=\sum_{\mathrm{i}=1}^{\mathbf{k}}(1+\mathrm{a} \operatorname{Cos} \theta)^{\frac{1}{2}} \operatorname{Cos} \theta / \sum_{i=1}^{\mathbf{k}}(1+\mathrm{a} \operatorname{Cos} \theta)^{\frac{1}{2}}
$$

Now if we assume $a$ to be reasonably small, the radicals of expression (1) may be expanded approximately as $(1+\mathrm{a} \operatorname{Cos} \theta)^{\frac{1}{2}} \simeq\left(1+\frac{1}{2} \mathrm{a} \operatorname{Cos} \theta\right)$, giving

$$
\begin{aligned}
& \stackrel{k}{\Sigma}(1+\mathrm{a} \operatorname{Cos} \theta)^{\frac{1}{2}} \operatorname{Cos} \theta \simeq \stackrel{k}{\Sigma}\left(\operatorname{Cos} \theta+\frac{1}{2} a \operatorname{Cos}^{2} \theta\right)= \\
& \mathrm{i}=1 \quad \mathrm{i}=1 \\
& \mathrm{ak} / 4
\end{aligned}
$$

and

$$
\sum_{i=1}^{k}(1+a \operatorname{Cos} \theta)^{\frac{1}{2}} \simeq \sum_{i=1}^{k}\left(1+\frac{1}{2} a \operatorname{Cos} \theta\right)=k
$$

by using elementary trigonometrical formulae. Hence from (1) we have $E(\bar{x})=a / 4$. A similar set of approximations yields $V(\bar{x})=V(\bar{y}) \simeq 1 /(8 N)$ as before. Combining these results and again assuming $\overline{\mathbf{x}}$ and $\bar{y}$ to have normal distributions implies that $\mathrm{X}^{2}$ has a non-central $\chi^{2}$ distribution with 2 df and non-centrality parameter $\mathrm{Na}^{2} / 2$.

Having established this result, to calculate the power of the test it only remains to evaluate the probability that such a non-central $\chi^{2}$ statistic exceeds the least significant value of the central (or usual) $\chi^{2}$ distribution. This was done by using an approximation of the non-central $\chi^{2}$ to the normal distribution due to Sankaran $(1959,1963)$ and referenced by Johnson and Kotz (1970; equation 23.4). This yields errors in the power estimates which are considerably smaller than would be important in most epidemiological applications of the results tabulated below.

\section{Results}

Table 1 shows the estimated power of the Edwards's test to detect seasonal variation of the simple harmonic form with amplitude $a$, and 
Table 1 Power (\%) of Edwards's test to detect simple harmonic seasonal variation with amplitude a for various sample sizes $N$

\begin{tabular}{rccccc}
\hline & \multicolumn{1}{l}{$a$} & & & \\
\cline { 2 - 6 } & $0 \cdot 01$ & $0 \cdot 02$ & 0.05 & $0 \cdot 10$ & $0 \cdot 20$ \\
\hline 50 & 5 & 5 & 5 & 7 & 13 \\
100 & 5 & 5 & 6 & 9 & 22 \\
250 & 5 & 5 & 7 & 15 & 50 \\
500 & 5 & 6 & 10 & 27 & 82 \\
1000 & 5 & 6 & 15 & 50 & 99 \\
2500 & 6 & 9 & 33 & 90 & 100 \\
5000 & 7 & 13 & 60 & 100 & 100 \\
10000 & 9 & 22 & 90 & 100 & 100 \\
25000 & 15 & 50 & 100 & 100 & 100 \\
50000 & 27 & 82 & 100 & 100 & 100 \\
100000 & 50 & 99 & 100 & 100 & 100 \\
\hline
\end{tabular}

Significance level $P=0.05$.

testing at the conventional $5 \%$ significance level. The results are given for various values of $a$ and the total sample size $\mathbf{N}$. It may be seen that when very substantial variation occurs (for example, $a=0.2$, implying a $20 \%$ difference in rates from the mean to the maximum) sample sizes of only 1000 or more will almost certainly give a significant test result. On the other hand, if only a $1 \%$ amplitude is envisaged $(a=0.01)$, even samples of 100000 cases will yield only a $50 \%$ chance of detecting the trend. For low values of both $a$ and $\mathrm{N}$, the power tends to the value $5 \%$; in such situations where a small study is investigating a very slight seasonal trend, significant results occur almost at random at approximately the same rate $(5 \%)$ as do Type I errors when the null hypothesis (no seasonal trend) is true. Table 2 shows the corresponding information for testing at the $1 \%$ significance level; because the probability of a Type I error (a significant result arising by chance when no seasonal trend is present) is much smaller here, the probability of a Type II error (failing to detect a seasonal trend which is actually present) is large, implying lower power at this more stringent level of significance as compared to Table 1.

Table 2 Power (\%) of Edwards's test to detect simple harmonic seasonal variation with amplitude a for various sample sizes $N$

\begin{tabular}{rccccc}
\hline & \multicolumn{6}{c}{ a } & \multicolumn{1}{c}{} \\
\cline { 2 - 6 } & $0 \cdot 01$ & 0.02 & $0 \cdot 05$ & $0 \cdot 10$ & $0 \cdot 20$ \\
\hline 50 & 1 & 1 & 1 & 2 & 4 \\
100 & 1 & 1 & 1 & 2 & 8 \\
250 & 1 & 1 & 2 & 5 & 27 \\
500 & 1 & 1 & 3 & 11 & 61 \\
1000 & 1 & 1 & 5 & 27 & 94 \\
2500 & 1 & 2 & 14 & 74 & 100 \\
5000 & 2 & 4 & 36 & 98 & 100 \\
10000 & 2 & 8 & 74 & 100 & 100 \\
25000 & 5 & 27 & 100 & 100 & 100 \\
50000 & 11 & 61 & 100 & 100 & 100 \\
100000 & 27 & 94 & 100 & 100 & 100 \\
\hline
\end{tabular}

Significance level $\mathbf{P}=\mathbf{0} \cdot 01$.
Note that the tables may be used in two ways. Firstly, one may determine directly the power of the test to detect seasonality of a certain amplitude with a sample of given size. Secondly, one may by interpolation find the sample size which would be required in order to achieve the desired power of detecting seasonality of given amplitude.

\section{Discussion}

It should be noted that the power of Edwards's test will in general be higher than that for the usual $\chi^{2}$ test for heterogeneity of rates between the $k$ time periods; this is because the Edwards's test takes into account the ordered structure of variation, whereas the usual $\chi^{2}$ test is equally sensitive to any pattern of variation. The difference in power between the two tests will be greater when more time intervals are used; this is because the usual $\chi^{2}$ statistic has (k-1) df, whereas the Edwards's $\chi^{2}$ statistic has $2 \mathrm{df}$, independently of $\mathrm{k}$; the critical value of $\chi^{2}$ is larger for higher values of $\mathbf{k}$, making the usual $\chi^{2}$ progressively weaker as $\mathbf{k}$ increases.

A further point is that the power is apparently not dependent on the value of $k$, the number of intervals into which the year is divided. This is not strictly true because one of the assumptions of the test is that the average rate for a time period is equal to the rate at the midpoint of that period, an assumption which is less exactly fulfilled when long time periods are used, and deviations from a linear trend within each time period become more pronounced. When shorter time intervals are used, it becomes possible to estimate the time of maximum rate more precisely, and to investigate the goodness-of-fit to and departures from the sinusoidal model in greater depth. On the other hand, the value of $\mathbf{k}$ may be determined to some extent by the sample size $\mathrm{N}$ and the way in which the data were collated; thus it would be unreasonable to carry out this test on 100 cases of a congenital abnormality using the week as the time interval, but a monthly grouping would probably be satisfactory; if the vital statistics source for this analysis presented the data by month, one would not have the option of using weeks in any case, but a recategorisation into quarterly data would be possible if necessary.

Potential users of the power results should be aware of a slight inconsistency in the definition of the amplitude of seasonal variation as used by different authors. Consider, for instance, the example of James (1976) who was concerned with determining the sample size required to be $80 \%$ 
certain of detecting 'a deviation of about 8 per cent' from a uniform distribution throughout the year of the birthdates of schizophrenics when testing at the usual 5\% significance level. The figure $8 \%$ had previously been suggested by Hare (1975), and was taken by James as indicating that the expected quarterly distribution of schizophrenic birthdates would be $25 \%, 27 \%, 25 \%$ and $23 \%$ (that is, a $2 \%$ swing from the percentage in an average quarter to the percentage in the quarter with the maximum 'rate', being proportionally an $8 \%$ deviation). Now observe that the $27 \%$ figure is in fact the rate for the whole of the quarter which contains the maximum, but is not equal to the rate at the exact date of the maximum, the latter will be slightly larger. If we again suppose that the maximum occurs at $0^{\circ}$, it may be shown by elementary calculus that in this case the instantaneous maximum for the rate is proportional to $27 \cdot 2 \%$, corresponding to an amplitude $a$ of approximately $8.9 \%$. (To derive this result, find, for example, the average value of $\operatorname{Cos} \theta$ in the range $-45^{\circ}$ to $+45^{\circ}$, the $90^{\circ}$ interval corresponding to a quarter of the year; this then yields the ratio between the average rate for the maximum quarter and the instantaneous maximum rate). Had a monthly interpretation of the postulated $8 \%$ deviation of Hare been used, the actual fluctuation would have been different again; it is clearly undesirable to allow the length of the time intervals to affect the analysis in this way, and when dealing with oscillations of such small magnitude (but typical of epidemiological data) such small differences in interpretation may have quite an impact on the power estimate. Tables 1 and 2 are for values of the amplitude $a$ which is the proportional difference between the average rate and the instantaneous maximum rate; all choices of time interval (months of the year, days of the week) may be examined using this general formulation of the problem.

A further common way in which the seasonal deviation is described is to give the ratio of the maximum to the minimum rate; in the terms of the notation of this paper, this ratio would be $(1+a) /(1-a)$.

It may be reasonably anticipated that the power results would be approximately true for situations in which the population at risk varies slightly. The appropriate test here would be that of Walter and Elwood (1975) or Edwards's test using case frequencies adjusted for the variable population at risk (although strictly speaking only the former is valid if the time intervals are also variable). In the numerical example of Walter and Elwood on the seasonality of anencephalus the $\chi^{2}$ test statistic was very similar using the two approaches, and this is empirical evidence that the two methods would have about the same power. The Walter-Elwood method is more correct, but the Edwards's test with corrected frequencies may be computationally slightly simpler in some analyses. A table of exact angles for the months of the year, and their trigonometric functions required in the Walter-Elwood test is given by Walter (1977).

Reprints from: S. D. Walter, Department of Epidemiology and Public Health, Yale University School of Medicine, 60 College Street, New Haven, Connecticut 06510, USA.

\section{References}

Edwards, J. H. (1961). The recognition and estimation of cyclic trends. Annals of Human Genetics, 25, 83-87.

Elwood, J. M. (1975). Seasonal variation in anencephalus in Canada. British Journal of Preventive and Socia建 Medicine, 29, 22-26.

Hare, E. H. (1975). Season of birth in schizophrenia and neurosis. American Journal of Psychiatry, 132, 1168-1171.

Hewitt, D., Milner, J., Csima, A., and Pakula, A. (1971). On Edwards' criterion of seasonality and a nonparametric alternative. British Journal of Preventive and Social Medicine, 25, 174-176.

James, W. H. (1976). The power of a test for seasonality of birth with reference to schizophrenia. The British Journal of Psychiatry, 129, 94-95.

Johnson, N. L., and Kotz, S. (1970). Continuous Univariate Distributions-2. Houghton Mifflin: Boston.

Sankaran, M. (1959). On the non-central chi-square distribution. Biometrika, 46, 235-237.

Sankaran, M. (1963). Approximations to the non-central chi-square distribution. Biometrika, 50, 199-204.

Symonds, R. L., and Williams, P. (1976). Seasonal variation in the incidence of mania. British Journal of Psychiatry, 129, 45-48.

Walter, S. D. (1977). Seasonality of mania: a reappraisal. British Journal of Psychiatry, 130 (In press).

Walter, S. D., and Elwood, J. M. (1975). A test for seasonality of events with a variable population at risk. British Journal of Preventive and Social Medicine, 29, 18-21. 